\title{
Naturally-occurring Wolbachia infection in Drosophila simulans that does not cause cytoplasmic incompatibility
}

\author{
ARY A. HOFFMANN*, DAVID CLANCY \& JACINTA DUNCAN \\ School of Genetics and Human Variation, La Trobe University, Bundoora, Victoria 3083, Australia
}

\begin{abstract}
Microbes of the genus Wolbachia are transmitted by their hosts via the maternal parent and are responsible for cytoplasmic incompatibility among insect populations. This phenomenon can result in Wolbachia spreading through natural populations as previously demonstrated in Drosophila simulans. Here we describe another Wolbachia infection in D. simulans that does not cause cytoplasmic incompatibility. This is a property of the Wolbachia rather than the nuclear background. The infection occurs at a low frequency in natural populations from eastern Australia. The infection shows perfect maternal transmission in the field and does not cause any detectable deleterious effects on its host. These findings suggest that the Wolbachia infection behaves like a neutral variant in populations. The infection may represent an evolutionary outcome of interactions between Wolbachia infections and their hosts.
\end{abstract}

Keywords: cytoplasmic incompatibility, Drosophila simulans, host-parasite evolution, maternal effects.

\section{Introduction}

Wolbachia are vertically-transmitted microbes that are widespread in insects. These microbes can cause cytoplasmic incompatibility in their host organisms. Infected males become reproductively incompatible with uninfected females or females carrying another Wolbachia strain, as shown in a range of organisms including mosquitoes (Yen \& Barr, 1973; Kambhampati et al., 1993), Drosophila (O'Neill et al., 1992; Rousset et al., 1992; Solignac et al., 1994), Nasonia (Breeuwer et al., 1992) and Tribolium (Wade \& Stevens, 1985). Wolbachia can also have other phenotypic effects on hosts including parthenogenesis in wasps (Stouthamer et al., 1993).

By causing cytoplasmic incompatibility, Wolbachia can spread in natural populations. Infected females have an advantage in populations that are polymorphic for the infection as they do not suffer incompatibility when they mate with infected or uninfected males (Caspari \& Watson, 1959; Fine, 1978; Hoffmann et al., 1990; Stevens \& Wade, 1990). Because the Wolbachia infection is almost always transmitted maternally, this advantage can result in an increase in the frequency of an infection. The spread of

\footnotetext{
${ }^{*}$ Correspondence.
}

(C) 1996 The Genetical Society of Great Britain.
Wolbachia will also be influenced by other factors, including any effects that microbes have on host fitness other than cytoplasmic incompatibility, and any leakage in the transmission of the infection between generations.

The behaviour of Wolbachia infections in natural populations has been studied most intensively in Drosophila, particularly in $D$. simulans. Two Wolbachia infections causing strong incompatibility have previously been described in this species. One of these infections (type R) has spread rapidly through California and also occurs in other areas (Hoffmann et al., 1990; Turelli \& Hoffmann, 1991). The other infection (type S) appears to be restricted to Pacific islands (Montchamp-Moreau et al., 1991). Strains with types $\mathrm{R}$ and $\mathrm{S}$ infections are bidirectionally incompatible (O'Neill \& Karr, 1990).

The distribution of the type $\mathbf{R}$ infection in natural D. simulans populations can be largely described in terms of two parameters: the level of incompatibility observed under field conditions and the efficiency whereby the microbe is transmitted from females to progeny (Hoffmann \& Turelli, 1988; Hoffmann et al., 1990; Turelli \& Hoffmann, 1991; Turelli et al., 1992). In addition, the spread of this infection can be influenced by deleterious effects on its host and by curing when larvae encounter naturally-occurring 
antibiotics (Stevens, 1989), although these effects have not been demonstrated under field conditions.

Previous work based on crosses between stocks indicate that neither the type $\mathrm{R}$ nor the type $\mathrm{S}$ infection is present in Australian populations of D. simulans (Hoffmann et al., 1990) even though Wolbachia do occur in Australian D. melanogaster (Hoffmann et al., 1994). However, we have recently detected another infection in $D$. simulans from Australia using Wolbachia-specific primers of an rDNA region (O'Neill et al., 1992) rather than the progeny tests we normally use to test for incompatibility. The newly-discovered infection has been designated as type A.

Here we show that the infection does not cause cytoplasmic incompatibility and that this is a property of the Wolbachia rather than the nuclear background. We also present data on the infection's distribution in natural populations of $D$. simulans. To understand why the infection is widespread in natural populations, we have considered its effects on host fitness and its maternal transmission efficiency in nature. Our findings suggest that the type A infection does not influence host fitness but shows perfect maternal transmission. The existence of infections with such properties has been predicted by theory (Turelli, 1994).

\section{Materials and methods}

\section{Detection and infection levels}

The newly-discovered Wolbachia infection was initially detected using the primers ' $76-99$ forward' (5'-TTGTAGCCTGCTATGGTATAACT-3') and '1012-994 reverse' (5'-GAATAGGTATGATTTTCATGT-3') of O'Neill et al. (1992) that amplify a $0.9 \mathrm{~kb}$ fragment of Wolbachia rDNA. As a positive control in PCR reactions, we used primers to amplify a single copy of a Drosophila nuclear gene, suppressor of forked (Langley et al., 1993), using the '5812-5829 forward' (5'-ATTGACTACCTGTCCCAT-3') and '6192-6174 reverse' (5'-ATAAGTAAATTCGGCGGTT- $3^{\prime}$ ) primers that yield a fragment of around $400 \mathrm{bp}$. This procedure produces a $0.9 \mathrm{~kb}$ band if Wolbachia are present that can be readily distinguished from the control band on an agarose gel.

The presence of the microorganism was confirmed by DAPI staining of embryos (O'Neill \& Karr, 1990). This staining method showed that the infection is localized in the outer cortex region, as is the case for other Wolbachia infections in D. simulans (O'Neill \& Karr, 1990) and for the Wolbachia infec- tion of Australian D. melanogaster (Hoffmann et al., 1994). We also used embryo staining to quantify levels of infection in a type A strain collected from Coffs Harbour in December 1993. For this purpose, we scored the density of bacteria in the outer cortex of embryos (Boyle et al., 1993). Wolbachia were visualized by epifluorescence microscopy of fixed embryos stained by indirect immunofluorescence. Embryos were collected and fixed following Hoffmann et al. (1994). They were then incubated at room temperature for $1 \mathrm{~h}$ using the primary, an antitype R Wolbachia IgM (supplied by Hroyuki Kose \& Tim Karr), diluted 1:2 in PBST (0.1 per cent Triton $\mathrm{X}-100$ in phosphate buffered saline) then rinsed three times in PBST. The embryos were incubated in the secondary, a goat anti-mouse IgM-TRITC (Southern Biotechnology Associates, Birmingham, AL 35226, U.S.A.) diluted 1:200 in PBST, for $1 \mathrm{~h}$ at room temperature in the dark, then rinsed three times in PBST. The nuclear stain DAPI $(1-2 \mu \mathrm{g}$ $\mathrm{mL}^{-1}$ for $8-12 \mathrm{~min}$ ) was used to assay their developmental stage, and the stained embryos were mounted as described in Hoffmann et al. (1994).

Only embryos displaying less than five nuclei were scored because densities can only be determined in young embryos (Boyle et al., 1993). The cortex region was scored to a depth of 11-12 microns from the surface over an area of 94 microns (along long axis) $\times 65$ microns. As well as examining 14 type A embryos (from an infected strain from Coffs Harbour), we also considered infection levels in 20 embryos from a type R strain from Ottawa (Hoffmann \& Turelli, 1988) and 13 embryos from infected D. melanogaster from Coffs Harbour (Hoffmann et al., 1994).

\section{Distribution and maternal transmission}

We used the PCR assay to examine the frequency of the type $\mathrm{A}$ infection along the east coast of Australia. In total, 1641 flies were collected from 12 sites along the coast (Fig. 1) between August 1993 and April 1994 by baiting with rotting fruit. D. simulans females were sorted from $D$. melanogaster females by abdominal banding patterns, cheek width (Gallo, 1973) and the appearance of the genital arch in male offspring. Flies were collected from two of the sites (Coffs Harbour, Gold Coast) on three separate occasions $(8 / 93,12 / 93,4 / 94)$.

Field females were frozen at $-5^{\circ} \mathrm{C}$ within 4 days of being collected, and eventually subjected to PCR to determine their infection status. If positive controls failed, extracts from females were retested. The infection status of all females collected from the 


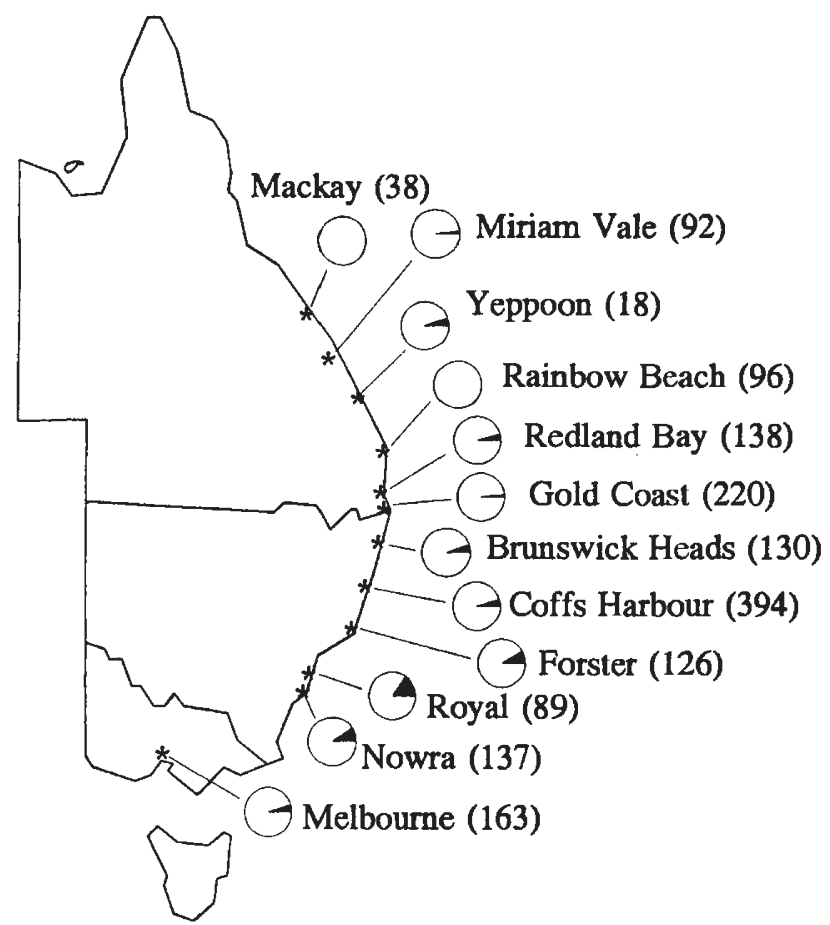

Fig. 1 Approximate location of Drosophila simulans collection sites in eastern Australia and the frequency of the type A Wolbachia infection. Shaded parts of the pie diagrams indicate infection frequencies determined by PCR analysis using Wolbachia-specific primers from the variable region of the $16 \mathrm{~S}$ gene. Numbers in brackets indicate sample sizes. Coffs Harbour and Gold Coast frequencies are based on three pooled samples. Frequencies and confidence intervals are: Melbourne: 0.052 $(0.028,0.089)$; Nowra: $0.008(0.059,0.149)$; Royal: 0.187 $(0.118,0.276)$; Forster: $0.096(0.075,0.154)$; Coffs Harbour: 0.051 (0.034, 0.072); Brunswick Heads: 0.057 (0.027, 0.107); Gold Coast: 0.018 (0.006, 0.041); Redland Bay: 0.045 (0.020, 0.086); Rainbow Beach: 0 (0, 0.047); Yeppoon: $0.059(0.017,0.252)$; Miriam Vale: $0.022(0.008$, 0.068); Mackay: $0(0,0.120)$.

field was determined. The use of PCR to determine infection levels in natural populations has previously been verified on type $\mathrm{R} D$. simulans where results were consistent with those obtained using progeny testing and DAPI staining (Turelli \& Hoffmann, 1995).

We tested for the efficiency of maternal transmission in field flies using the method of Hoffmann et al. (1990), except that the infection was detected by PCR assay rather than progeny testing. We used females from Coffs Harbour collected on 12/93, 4/94 and $8 / 94$. We tested these for infection status with the PCR assay after they had produced eggs. We identified 21 infected females in this manner. Between 15 and 40 progeny from each of the females were tested for infection status using the PCR assay to determine transmission rates.

\section{Incompatibility and fecundity}

Four experiments were undertaken to investigate the effects of the infection on incompatibility. In each experiment, pairs of males and females (2-3 days posteclosion) were set up in vials containing a spoon with $5 \mathrm{~mL}$ of a blackcurrant medium (61 per cent blackcurrant syrup, 5 per cent agar) covered with a live yeast suspension. Eggs were removed after $24 \mathrm{~h}$ and hatch rates were scored after $24 \mathrm{~h}$ at $25^{\circ} \mathrm{C}$. Spoons with less than 10 eggs were not considered. In all experiments, type A flies were obtained from an infected strain collected from Coffs Harbour in December 1993.

In the first experiment, we examined the ability of type A males to cause incompatibility when mated with uninfected $D$. simulans. To provide controls, crosses were carried out between uninfected females and uninfected males or type $\mathbf{R}$ males. The former were expected to be compatible and the latter were expected to be incompatible. We set up a large number of replicates (93) for crosses with uninfected males and type A males so that we could detect low levels of incompatibility.

In the second experiment, we tested incompatibility in type A females. Females were crossed to type A males, strains of type $\mathrm{R}$ males from Riverside (Hoffmann et al., 1986) or Ottawa (Hoffmann \& Turelli, 1988) and type $S$ males from a Hawaii strain. The infected Hawaii strain was collected in 1991 from Volcano.

The final two experiments tested the inheritance of type A incompatibility. Reciprocal crosses were carried out between the Riverside strain and the type A strain from Coffs Harbour. In the third experiment, $F_{1}$ males from the reciprocal crosses were mated to uninfected females. Crosses between Riverside males and uninfected females were also set up. In the fourth experiment, Riverside males were crossed to $F_{1}$ females from the reciprocal crosses. Riverside males were also crossed to type $\mathrm{A}$ females and uninfected females.

Apart from causing incompatibility, Wolbachia may have deleterious fitness effects on its host. In the case of type $\mathrm{R} D$. simulans, infected females have a lower fecundity than uninfected females, at least under laboratory conditions (Hoffmann et al., 1990). Deleterious effects have also been associated with Wolbachia infections of Tribolium (Stevens \& Wade, 1990). To test if fecundity costs were associated with the type $\mathrm{A}$ infection, we cured an infected 
line from Coffs Harbour by culturing it for a generation on medium supplemented with 0.03 per cent tetracycline (Hoffmann et al., 1986), followed by a generation on unsupplemented medium to remove any effects of tetracycline exposure. The infected and uninfected lines differ in their infection status but not in their nuclear background. The fecundity of the two lines was measured following Hoffmann et al. (1994). For each line, 71 pairs of males and females (3-4 days old) were set up in vials with a small spoon containing laboratory medium covered with a live yeast suspension. Spoons were replaced daily for three days and egg numbers were summed to give an estimate of fecundity for each pair of flies.

\section{Results}

\section{Infection levels}

We anticipated that if microbe numbers were correlated with incompatibility levels, numbers for the type $\mathrm{A}$ infection should be lower than for the type $\mathrm{R}$ infection and the $D$. melanogaster infection. We therefore undertook one-tailed tests to compare the means. Because the two comparisons between the type $\mathrm{A}$ infection and either type $\mathrm{R}$ or $D$. melanogaster were not orthogonal, we corrected for the number of comparisons (2) using the Bonferonni technique. Counts between the $\mathrm{R}$ and $\mathrm{A}$ infections differed significantly at the 5 per cent level $\left(F_{1,32}=4.79\right)$, and numbers were substantially higher for the $\mathrm{R}$ infection. However, differences between type $\mathrm{A}$ and $D$. melanogaster were in the wrong direction (Fig. 2).

\section{Distribution and maternal transmission}

The A infection is widespread along the east coast of Australia and present at a low frequency in most populations (Fig. 1). Contingency tests indicated no differences in infection frequencies in the three collections from Coffs Harbour $(G=0.314$, d.f. $=2)$ and the two largest collections from the Gold Coast (Fisher's exact test, $P=0.63$ ). Data were therefore pooled from these sites before making comparisons between populations.

To compare infection frequencies among the populations, collections north of Rainbow Beach were pooled to overcome the problem of having too many expected values less than five. Infection frequencies differed significantly among populations by a contingency test $\left(G_{9}=40.24, P<0.001\right)$. Population differences were also significant if the pooled localities were excluded $\left(G_{8}=40.78, \quad P<0.001\right)$.

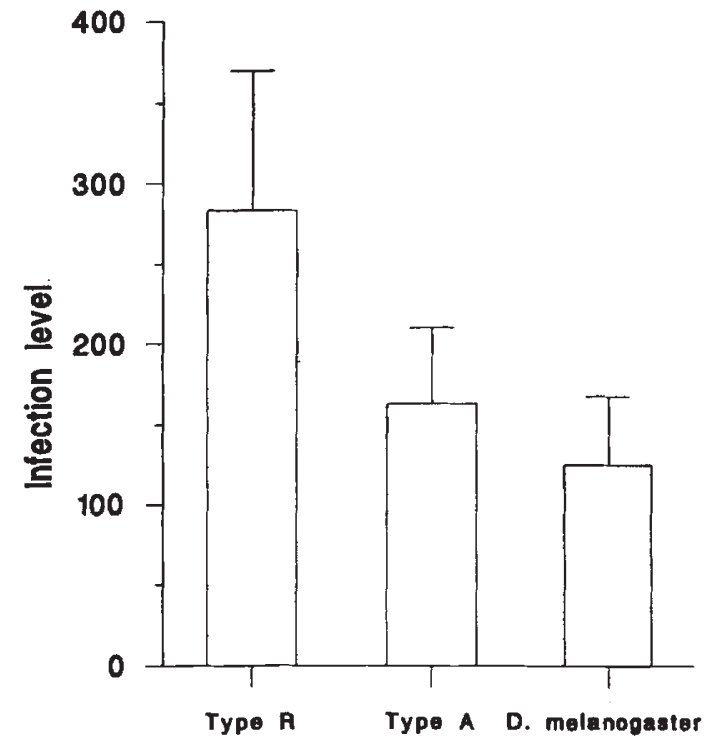

Fig. 2 Levels of infection in embryos from type A or type $\mathrm{R}$ Drosophila simulans or from infected $D$. melanogaster. Numbers represent Wolbachia counts in an area of 6110 $\mu \mathrm{m}^{2}$ from the outer cortical region of embryos. Means and standard deviations (error bars) are based on 14 (type A), 20 (type $\mathrm{R}$ ) or 13 (D. melanogaster) embryos.

Confidence limits for infection frequencies were determined following Zar (1984) and are given in the legend of Fig. 1. These indicate that the main difference between sites is the high frequency of infection in the Royal National Park, just south of Sydney. There is no obvious geographical pattern which suggests that infection frequencies are not related to climatic variables that vary latitudinally.

In the experiments on maternal transmission efficiency, all 572 of the progeny that were tested carried the Wolbachia infection. When 95 per cent confidence intervals are computed, the upper bound for the proportion of uninfected progeny produced by infected mothers is 0.01 . Maternal transmission therefore seems to be perfect or near-perfect, in contrast to transmission of the type $\mathrm{R}$ infection of $D$. simulans (Hoffmann et al., 1990) and a Wolbachia infection in D. melanogaster (A. A. Hoffmann \& J. Duncan, unpublished data) where 3-9 per cent of progeny from infected females do not carry the infection.

\section{Incompatibility and fecundity}

Hatch rates from crosses were compared with nonparametric Kruskal-Wallis tests because the data had skewed distributions. These tests indicated significant differences $(P<0.001)$ among crosses in all four experiments. Differences between individual 
crosses were examined using the STP approach for unplanned nonparametric comparisons outlined in Sokal \& Rohlf (1981).

Results from experiments 1 and 2 indicate that the infection did not cause incompatibility. Males from $D$. simulans strains infected with Wolbachia are normally incompatible with females from uninfected strains (Hoffmann et al., 1986; O'Neill \& Karr, 1990) and this is evident in the crosses with type $\mathrm{R}$ and type $\mathrm{S}$ males (Table 1). However, hatch rates in crosses between uninfected individuals are indistinguishable from those in crosses between type A males and uninfected females, despite the fact that we set up 93 replicates in one of the experiments. Males with the A infection therefore behaved like uninfected individuals. Females from strains with the A infection also behaved like those from uninfected strains. These females were incompatible with both type $\mathrm{R}$ and type $\mathrm{S}$ males, but compatible with males that carried the A infection (Table 1). We have obtained similar results with three other infected $D$. simulans strains from eastern Australia (data not given).

The $F_{1}$ data from experiments 3 and 4 indicate that the inability of type A males to cause incompatibility resides in the genome of Wolbachia or another cytoplasmic factor rather than in the genome of its host. When $F_{1} s$ were obtained from reciprocal crosses between strains with $\mathrm{R}$ and $\mathrm{A}$ infections, males behaved the same as their maternal infection type when they were crossed to uninfected females (experiment 3, Table 1). The susceptibility of females to incompatibility caused by the $\mathrm{R}$ infection is also inherited maternally because $F_{1}$ females behaved like their maternal parent (experiment 4). We have carried out successive generations of backcrossing to an $\mathrm{R}$ strain to demonstrate that the type A infection is inherited via the maternal parent. For instance, after the progeny from a cross between $A$ females and $\mathrm{R}$ males were backcrossed to $\mathrm{R}$ males for three generations, the mean hatch rate from matings between backcross males and uninfected females was $0.843(\mathrm{SD}=0.147, N=29)$ compared with a mean hatch rate for controls (uninfected $x$ uninfected) of $0.869(\mathrm{SD}=0.192, N=30)$.

The fecundity data indicated that the infection was not associated with deleterious effects. The mean number of eggs produced by the infected line $(\bar{x}=273.8, \mathrm{SD}=61.1, N=71)$ did not differ significantly from the mean produced by the cured line $(\bar{x}=265.2, \mathrm{SD}=80.5, N=71) . D$. simulans females carrying the A infection therefore did not suffer a detectable decrease in fecundity under laboratory conditions such as found in type $\mathrm{R}$ strains (Hoffmann et al., 1990). We have also failed to find deleterious effects for other traits related to fitness, including egg-to-adult development time, body size, larval viability and the resistance of adults and eggs to environmental stresses.

\section{Discussion}

The Australian Wolbachia infection of $D$. simulans does not appear to cause incompatibility under

Table 1 Hatch rates in four experiments involving crosses between a Wolbachia-infected Drosophila simulans strain from Australia and other uninfected and infected $D$. simulans strains

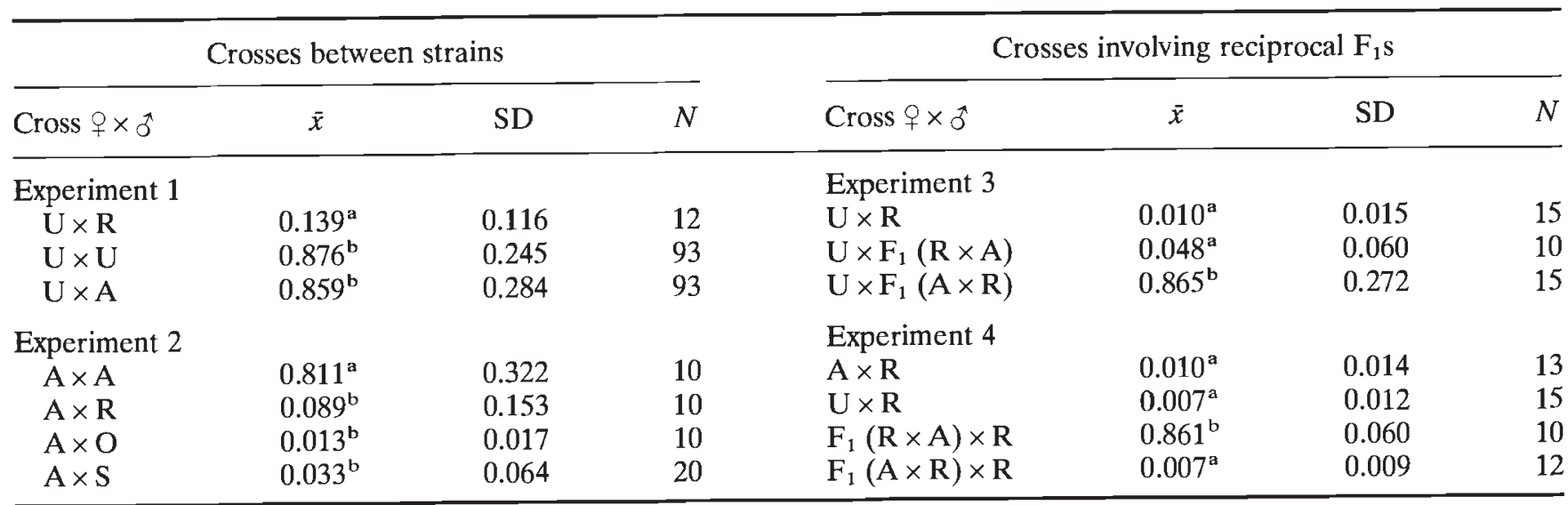

Abbreviations: $\mathrm{U}$ is an uninfected $D$. simulans strain from Melbourne; $\mathrm{R}$ is an infected type $\mathrm{R} D$. simulans strain from Riverside; $\mathrm{O}$ is an infected type $\mathrm{R} D$. simulans strain from Ottawa; A is an infected $D$. simulans strain from Coffs Harbour, Australia, $\mathrm{S}$ is an infected $D$. simulans strain from Hawaii; and $\mathrm{F}_{1}$ refers to $\mathrm{F}_{1}$ males or females produced from reciprocal crosses between $R$ females and $A$ males $(R \times A)$ or between $A$ females and $R$ males $(A \times R)$.

Means followed by the same letter do not differ significantly by the nonparametric STP procedure. 
laboratory conditions. Power tests indicate that we could have detected incompatibility levels of around 4 per cent or more in the comparison of crosses involving uninfected males and type A males, so if any incompatibility is present it must be extremely weak. Because studies with the type $\mathrm{R}$ infection of $D$. simulans indicate that incompatibility levels in the field tend to be lower than in the laboratory (Hoffmann et al., 1990), the type A infection is also unlikely to cause incompatibility under field conditions. Low levels of incompatibility have previously been found in laboratory studies on $D$. melanogaster (Hoffmann, 1988; Holden et al., 1993) and Giordano et al. (1995) have recently described a Wolbachia infection in D. mauritiana that does not appear to cause incompatibility. Wolbachia therefore may have variable effects on cytoplasmic incompatibility in Drosophila and probably in other insects as well.

Expected changes in the frequency of an infection in a population have previously been modelled with simple equations (Caspari \& Watson, 1959; Fine, 1978; Hoffmann et al., 1990; Turelli et al., 1992). These equations have successfully predicted the spread of type R Wolbachia in Californian populations of $D$. simulans (Turelli \& Hoffmann, 1991). In the absence of incompatibility, the frequency of a Wolbachia infection $(p)$ in an isolated population at generation $t+1$ is given by the equation

$p_{t+1}=\frac{p_{t}(1-\mu)\left(1-s_{f}\right)}{1-s_{f} p_{t}}$,

where $1-s_{\mathrm{f}}$ is the relative fecundity of infected females and $\mu$ is the proportion of progeny produced by an infected female that is no longer infected. The $\mu$ term is included in this equation because previous work has shown that maternal transmission of the infection is not perfect (i.e. $\mu>0$ ) (Hoffmann et al., 1990). It has also been shown previously that Wolbachia infections can have deleterious effects on the fecundity of $D$. simulans (Hoffmann \& Turelli, 1988; Hoffmann et al., 1990; Nigro \& Prout, 1990). In the equation, $p_{t+1}$ will be less than $p_{t}$ if $\mu$ is greater than 0 and/or if $s_{\mathrm{f}}$ falls between 0 and 1 . If the type $\mathrm{A}$ infection is to persist in populations, maternal transmission must therefore be perfect in the field and there should be no deleterious effects on fecundity (or other fitness components). On the other hand, the type A infection will only spread if infected females have a fitness advantage (i.e. $s_{\mathrm{f}}<0$ ).

The results of this paper indicate that $\mu$ is likely to be 0 or very close to 0 . In addition, $s_{\mathrm{f}}$ appears to be near 0 in the laboratory. A power test indicates that we could have detected a fecundity effect of around 7 per cent. If any deleterious effects on host fecundity are present, they must be small. The type A infection could therefore persist in natural populations of $D$. simulans even without causing incompatibility, behaving as a neutral variant whose frequency is determined by random processes. Differences in infection frequencies among $D$. simulans populations along the east coast of Australia may reflect random events, such as the recolonization of an area following unfavourable conditions.

It has previously been postulated that there is an association between levels of incompatibility and the density of Wolbachia in cells (Breeuwer \& Werren, 1993). High densities of Wolbachia may be associated with high levels of incompatibility. Supporting evidence has come from Wolbachia infections in different strains of Nasonia (Breeuwer \& Werren, 1993) and D. melanogaster (Solignac et al., 1994) and in comparisons of Wolbachia numbers in $D$. simulans vs. D. melanogaster (Boyle et al., 1993). However, our data indicate that embryo infection levels are not the sole cause of incompatibility. Infection levels in the type A strain were similar to those in $D$. melanogaster. Because infected D. melanogaster show partial incompatibility (Hoffmann et al., 1994), the results indicate that levels of incompatibility in Drosophila are not strictly correlated with the density of Wolbachia in embryos. The intermediate density of Wolbachia in type A embryos also indicates that maternal transmission efficiency is not necessarily related to Wolbachia density as postulated elsewhere (Turelli, 1994) because maternal transmission of the type $\mathrm{R}$ infection is less efficient than that of the type A infection.

How might this infection have become widely distributed in Australian D. simulans populations? The reason why the $\mathrm{R}$ Wolbachia infection has spread in California is because it causes incompatibility when infected males mate with uninfected females. However, evolutionary changes in the genome of Wolbachia and in the genome of their hosts do not necessarily favour increasingly higher levels of incompatibility. Instead, theoretical models (Turelli, 1994) show that infections associated with lower levels of incompatibility can be favoured when they have a higher rate of maternal transmission and when they have a reduced deleterious effect on their host organism.

The A infection of $D$. simulans may represent the outcome of such an evolutionary process. Once the A infection had spread and supplanted the ancestral type, it may have decreased in frequency because of random events, unidentified deleterious effects on 
the host or perhaps occasional curing of larvae exposed to antibiotics produced by naturally-occurring microorganisms. The low frequency of this infection in extant $D$. simulans populations from Australia could therefore reflect a relict distribution of an infection that was once widespread and predates the colonization of Australia by $D$. simulans because infected $D$. simulans strains that do not cause incompatibility have recently been found at a low frequency in populations from North and South America (Turelli \& Hoffmann, 1995).

Other explanations also need to be considered. For instance, infection levels may represent an equilibrium between environmental curing and reinfection by parasites carrying Wolbachia. It is possible that infected individuals become completely cured after exposure to natural antibiotics even though environmental curing does not appear to influence maternal transmission of the A infection. Reinfection rates mediated by parasites have not been measured. However sympatric sibling species such as $D$. melanogaster and $D$. simulans that share the same parasites do not share the same infections (e.g. Hoffmann et al., 1990), suggesting that parasite infection rates may be low. The presence of Wolbachia in Drosophila parasites such as mites and parasitic wasps could be screened to provide evidence on this hypothesis.

\section{Acknowledgements}

We thank Ross Crozier, Ben Oldroyd and Michael Turelli for comments on an earlier draft. This work was supported by the Australian Research Council.

\section{References}

BOYLE, L., O'NEILL, S. L., ROBERTSON, H. AND KARR, T. L. 1993. Horizontal transfer of Wolbachia pipientis by microinjection of egg cytoplasm: infection levels and the expression of cytoplasmic incompatibility in Drosophila. Science, 260, 1796-1799.

BREEUWER, J. A. J. AND WERREN, J. H. 1993. Cytoplasmic incompatibility and bacterial density in Nasonia vitripennis. Genetics, 135, 565-574.

BREEUWER, J. A. J., STOUTHAMER, R., BARNS, S. M., PELletier, D. A., WeIsBurg, W. G. AND WERREN, J. H. 1992. Phylogeny of cytoplasmic incompatibility microorganisms in the parasitoid wasp genus Nasonia (Hymenoptera, Pteromalidae) based on $16 \mathrm{~S}$ ribosomal sequences. Insect Mol. Biol., 1, 25-36.

CASPAR1, E. AND WATSON, G. s. 1959. On the evolutionary importance of cytoplasmic sterility in mosquitoes. Evolution, 13, 568-570.

FINE, P. E. M. 1978. On the dynamics of symbiont-dependent cytoplasmic incompatibility in culicine mosquitoes.
J. Invert. Pathol., 30, 10-18.

GALlo, A. J. 1973. Morphological distinction between female Drosophila melanogaster and female $D$. simulans. Cienc. cult. San Paulo, 25, 341-345.

GIORDANO, R., O'NEILL, S. L. AND ROBERTSON, H. M. 1995. Wolbachia infections and the expression of cytoplasmic incompatibility in Drosophila sechellia and D. mauritiana. Genetics, 140, 1307-1317.

HOFFMANN, A. A. AND TURELL1, M. 1988. Unidirectional incompatibility in Drosophila simulans: inheritance, geographic variation and fitness effects. Genetics, 119, $435-444$.

HoffmanN, A. A., TUREll1, M. AND Simmons, G. M. 1986. Unidirectional incompatibility between populations of Drosophila simulans. Evolution, 40, 692-701.

hoffmanN, A. A., TURELli, M. AND haRshman, L. G. 1990. Factors affecting the distribution of cytoplasmic incompatibility in Drosophila simulans. Genetics, 126, 933-948.

HOFFMANN, A. A., ClanCy, D. J. AND MERTON, E. 1994. Cytoplasmic incompatibility in Australian populations of Drosophila melanogaster. Genetics, 136, 993-999.

HOLDEN, P. R., JONES, P. AND BROOKFIELD, J. F. Y. 1993. Evidence for a Wolbachia symbiont in Drosophila melanogaster. Genet. Res., 62, 23-29.

KAMBHAMPATl, S., RAl, K. S. AND BURGUN, S. J. 1993. Unidirectional cytoplasmic incompatibility in the mosquito, Aedes albopictus. Evolution, 47, 673-677.

LANGLEY, C. H., MACDONALD, J., MIYASHITA, N. AND AGUADE, M. 1993. Lack of correlation between interspecific divergence and intraspecific polymorphism at the suppressor of forked region in Drosophila melanogaster and Drosophila simulans. Proc. Natl. Acad. Sci. U.S.A., 90, 1800-1803.

MONTCHAMP-MOREAU, C., FERVEUR, J.-F. AND JACOUES, M. 1991. Geographic distribution and inheritance of three cytoplasmic incompatibility types in Drosophila simulans. Genetics, 129, 399-407.

NIGRO, L. AND PROUT, T. 1990. Is there selection on RFLP differences in mitochondrial DNA? Genetics, 125, $551-555$.

O'NElLL, S. L. AND KARR, T. L. 1990. Bidirectional incompatibility between conspecific populations of Drosophila simulans. Nature, 348, 178-180.

O'NEILL, S. L., GIORDANO, R. COLBERT, A. M. E., KARR, T. L. AND ROBERTSON, H. M. 1992. 16S rRNA phylogenetic analysis of the bacterial endosymbionts associated with cytoplasmic incompatibility in insects. Proc. Natl. Acad. Sci. U.S.A., 89, 2699-2702.

Rousset, F., VAutrin, D. AND SolıGNAC, M. 1992. Molecular identification of Wolbachia, the agent of cytoplasmic incompatibility in Drosophila simulans, and variability in relation with host mitochondrial types. Proc. R. Soc. B, 247, 163-168.

SOKAL, R. R. AND ROHLF, F. J. 1981. Biometry, 2nd edn. Freeman, New York.

SOLIGNAC, M., VAUTRIN, D. AND ROUSSET, F. 1994. Widespread occurrence of the proteobacteria Wolbachia and partial incompatibility in Drosophila melanogaster. C.r. 
Acad. Sci. Paris, Ser. III, Sciences de la vie, 317, 461-470. STEVENS, L. 1989. Environmental factors affecting reproductive incompatibility in flour beetles, genus Tribolium. J. Invert. Pathol., 53, 78-84.

STEVENS, L. AND WADE, M. J. 1990. Cytoplasmically inherited reproductive incompatibility in Tribolium flour beetles: the rate of spread and effect on population size. Genetics, 124, 367-372.

STOUTHAMER, R., BREeUWER, J. A. J., LUCK, R. F. AND WERREN, J. H. 1993. Molecular identification of microorganisms associated with parthenogenesis. Nature, 361, $66-68$.

TURELL1, M. 1994. Evolution of incompatiblity-inducing microbes and their hosts. Evolution, 48, 1500-1513.

TURELL1, M. AND hOFFMANN, A. A. 1991. Rapid spread of an inherited incompatibility factor in California Drosophila. Nature, 353, 440-442.
TURELli, M. AND hofFMANN, A. A, 1995. Cytoplasmic incompatibility in Drosophila simulans: dynamics and parameter estimates from natural populations. Genetics, 140, 1319-1338.

TURELll, M., HOFFMANN, A. A. AND MCKECHNIE, S. W. 1992. Dynamics of cytoplasmic incompatibility and mtDNA variation in natural Drosophila simulans populations. Genetics, 132, 713-723.

WADE, M. J. AND STEVENS, L. 1985. Microorganism mediated reproductive isolation in flour beetles (genus Tribolium). Science, 227, 527-528.

YEN, J. H. AND BARR, A. R. 1973. The etiological agent of cytoplasmic incompatibility in Culex pipiens. J. Invert. Pathol., 38, 409-418.

ZAR, J. H. 1984. Biostatistical Analysis, 2nd edn. PrenticeHall, Englewood Cliffs, NJ. 